\title{
Type 2 Myocardial Infarction: A Geriatric Population- based Model of Pathogenesis
}

\author{
Alain Putot ${ }^{1,4, *}$, Melanie Jeanmichel ${ }^{2}$, Frederic Chague $^{2}$, Patrick Manckoundia ${ }^{1,3}$, Yves Cottin ${ }^{2,4}$, \\ Marianne Zeller $^{4}$
}

${ }^{1}$ Geriatric Department, University Hospital of Dijon Bourgogne, France.

${ }^{2}$ Cardiology Department, University Hospital of Dijon Bourgogne, France.

${ }^{3}$ INSERM U1093 Cognition Action Plasticite, Universite de Bourgogne Franche Comte, France.

${ }^{4}$ Physiopathologie et Epidémiologie Cerebro-Cardiovasculaires (PEC2), Universite de Bourgogne Franche

Comte, France.

[Received March 3, 2019; Revised March 29, 2019; Accepted April 5, 2019]

\begin{abstract}
Distinction between type 2 myocardial infarction (T2MI), defined as an imbalance between oxygen supply and demand without atherothrombosis, and type 1 myocardial infarction (T1MI), due to plaque disruption, is often a clinical challenge in frail elderly patients. We aimed to identify the characteristics and underlying causes of T2MI using a comprehensive geriatric approach. From a multicentre population-based prospective study in coronary care units, we adjudicated 4572 consecutive patients hospitalized for an acute T1MI or T2MI, according to the $3^{\text {rd }}$ universal definition and a prespecified geriatric model of T2MI pathogenesis. In total, $3710(81 \%)$ had T1MI and $862(19 \%)$ T2MI. Patients with T2MI were 10 y older (77 vs $67 \mathrm{y}, p<0.001)$, more frequently female $(44 \mathrm{vs} 26 \%, p<0.001)$ and had more frequent comorbidities. In multivariate analysis, acute heart failure, tachycardia and C-reactive protein elevation at admission were associated with a higher risk of T2MI vs T1MI, whereas chest pain, troponin I peak $>10 \mu \mathrm{g} / \mathrm{L}$ and ST-segment elevation were associated with a lower risk. Underlying mechanisms leading to T2MI highlighted 3 main patterns: 1) Age-related physiological cardiovascular decline 2) chronic predisposing factors including chronic anaemia (10\%) and severe aortic stenosis $(7 \%), 3)$ acute triggering factors, the most common being acute infection (39\%), mainly respiratory tract infection, followed by tachyarrhythmia $(13 \%)$ and acute heart failure $(10 \%) .122(14 \%)$ patients had combined predisposing and triggering conditions for T2MI. In our large population-based survey of T2MI, chronic anaemia and severe aortic stenosis increased predisposition to T2MI and acute respiratory infection was by far the most frequent trigger. Our data shed new light on the age-related pathophysiological basis for discrepancies in oxygen supply and demand leading to MI.
\end{abstract}

Key words: type 2 myocardial infarction, anemia, respiratory tract infection, aortic stenosis, tachyarrhythmia pathophysiology

Type 2 myocardial infarction (T2MI), newly redefined by the fourth universal definition of myocardial infarction (MI) [1], is an emerging clinical condition in older patients resulting from a mismatch between supply and demand of myocardial oxygen in the absence of atherothrombosis. This model was initially based on autopsy data which highlighted the lack of thrombi in the coronary arteries of $31 \% \mathrm{MI}$ deaths [2], and contemporary imaging studies demonstrating the heterogeneity in underlying causes of acute MI [3]. However, pinpointing the clinical conditions leading to T2MI has proven difficult, and distinguishing between myocardial injury, type $1 \mathrm{MI}$ (T1MI) and T2MI remains a major challenge in routine clinical practice [4]. Because coronary

*Correspondence should be addressed to: Dr. Alain Putot, Geriatric Department, University Hospital of Dijon Bourgogne, 2 rue Jules Violle, 21033 Dijon Cedex. France. Email: alain.putot@chu-dijon.fr.

Copyright: () 2019 Putot A et al. This is an open-access article distributed under the terms of the Creative Commons Attribution License, which permits unrestricted use, distribution, and reproduction in any medium, provided the original author and source are credited. 
angiography is only rarely performed in frail elderly patients, there is a need for clinical markers for every-day distinction between T2MI and T1MI. In contrast with T1MI, corresponding to spontaneous plaque rupture or erosion and resulting in thrombus formation, T2MI pathogenesis is complex and multifactorial: multiple and heterogeneous situations may co-exist and lead to a myocardial supply/demand mismatch. Criteria for defining T1MI have varied markedly form study to study. As a consequence, variations in the prevalence of T2MI ranging from $1.6 \%$ to $74 \%$ have been reported in the literature $[5,6]$. Although several specific clinical criteria have been proposed for the practical application of the universal definition $[7,8]$, only few prospective data are available to date to support these criteria $[9,10]$ and investigation of the underlying mechanisms is poor. In particular, the potential combination of acute and chronic clinical conditions promoting the development of oxygen mismatch has never been explored.

From a large multicentre contemporary prospective survey, we aimed to comprehensively describe the factors leading to T2MI and to propose a new model of etiologic classification using a geriatric approach of pathogenesis.

\section{MATERIALS AND METHODS}

\section{Patients}

Characteristics of the French regional obseRvatoire des Infarctus de Côte d'Or (RICO) survey have previously been described [11]. Briefly, RICO is an ongoing survey that prospectively collects data from patients hospitalized for acute MI in the cardiology intensive care units of teaching hospitals, general hospitals, and private clinics receiving acute MI emergencies for one region of eastern France. From October 1st, 2012 to March 31st, 2017, all consecutive patients admitted for type 1 or type 2 MI within 24 hours after symptom onset were included in the present study. T1MI or T2MI was prospectively adjudicated according to third universal definition [12]. Patients with myocardial injury, Takotsubo cardiomyopathy, type 3, 4 or 5 MI were excluded from the analysis.

The present study complied with the Declaration of Helsinki and was approved by the Ethics Committee of Dijon University Hospital. Each patient gave written consent before participation.

\section{Data collection}

Demographic data, cardiovascular risk factors and history were collected for all patients as were on-admission ECG, clinical and biological data, as described previously [11]. Length of intensive care unit (ICU) stay and GRACE score for mortality risk [13] were also included [13]. Blood samples were taken on admission to measure haemoglobin and C-reactive protein levels, plasma NT proBNP, Low Density Lipoprotein (LDL) cholesterol and serum creatinine. The estimated glomerular filtration rate was calculated using the Chronic Kidney DiseaseEPIdemiology Collaboration formula (CKD-EPI). Cardiac troponin I peak (Dimension Vista LOCI assay) [14] was assessed by sampling every 8 hours during the first 2 days after admission. Left ventricle ejection fraction was measured by echocardiography. Coronary angiography data, including normal and non-obstructive coronary artery (MINOCA) [15] rates and rate of reperfusion (percutaneous coronary intervention, coronary bypass surgery and thrombolysis) were also collected.

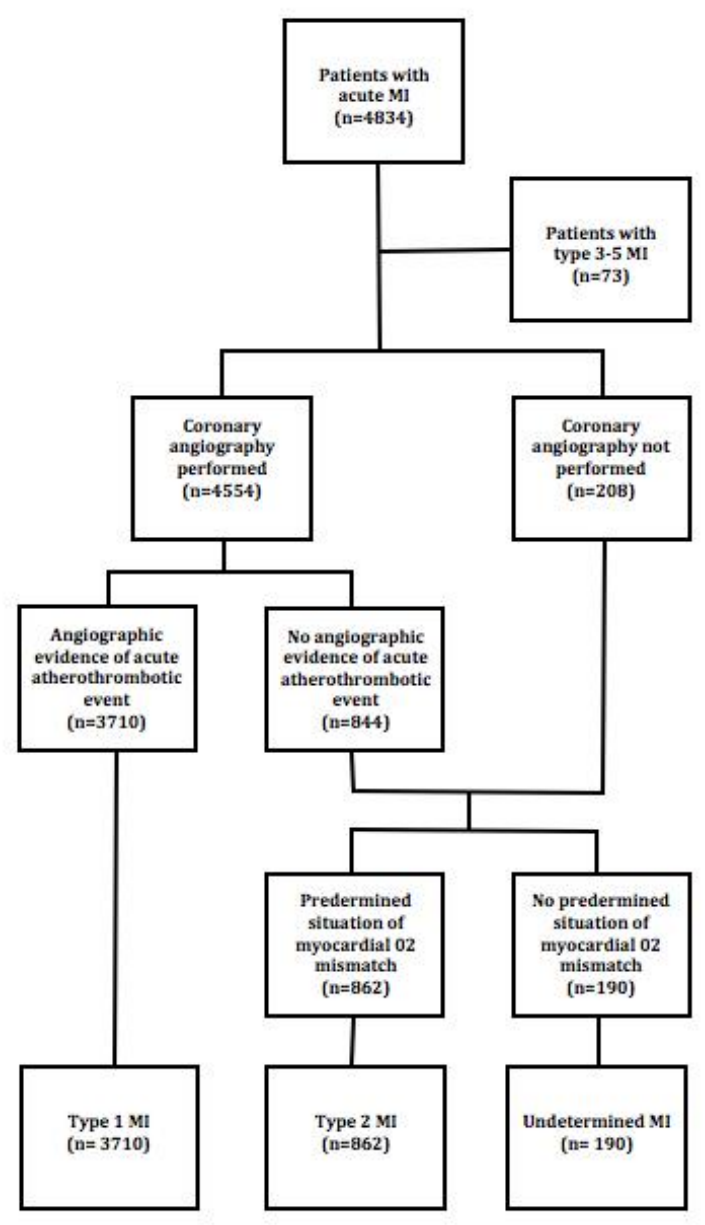

Figure 1. Flow chart.

\section{Identification of Type 2 MI cases}

Each case was reviewed and adjudicated by two independent reviewers (one cardiologist and one internist) with a $85 \%$ inter-observer agreement. Any discrepancies 
were resolved by consensus after an in-depth review of the patient's medical records. MI were defined according to the third universal definition established in 2012, i.e. rise of cardiac troponin above the $99^{\text {th }}$ percentile upper reference limit and with at least one of the following: symptoms of ischemia, new significant ST changes or new left bundle branch block, development of pathological Q waves [12]. T1MI was defined as MI related to ischemia due to a primary coronary event such as spontaneous plaque erosion or rupture, intraluminal thrombus or coronary dissection. T2MI was defined by the absence of evidence of plaque rupture at coronary angiography when available and at least one of the conditions listed below at the onset of MI symptoms [7,8]. Study flow chart and T1MI/T2MI adjudication criteria are presented in Figure 1. Conditions were identified according to previous publications, covering the whole spectrum of T2MI cases and classified as chronic (predisposing) or acute (precipitating) factors.

\section{Predisposing factors}

- Severe aortic stenosis [10], diagnosed by doppler echocardiography and defined according to current guidelines [16]

- Hypertensive cardiomyopathy [7], defined as essential hypertension with a systolic blood pressure (BP) $>160 \mathrm{mmHg}$ and concomitant left ventricular hypertrophy identified by echocardiography and/or ECG

- Thyrotoxicosis [10], defined as documented clinical symptoms of hyperthyroidism associated with elevated peripheral hormones

- Chronic severe anaemia [7], defined as haemoglobin $<5.5 \mathrm{mmol} / \mathrm{L}$ for men and $<5.0 \mathrm{mmol} / \mathrm{L}$ for women (measured on admission) and/or the need to use blood products.

\section{Precipitating factors}

- Active bleeding [8], defined as acute external bleeding with haemoglobin rate $<5.5 \mathrm{mmol} / \mathrm{L}$ for men and $<5.0 \mathrm{mmol} / \mathrm{L}$ for women and/or the need to use blood products.

- Acute respiratory failure [7], defined as clinical signs lasting $\geq 20$ minutes with an arterial oxygen tension $<60 \mathrm{mmHg}$, from non-cardiogenic and non-infectious causes;

- Severe acute heart failure occurring prior to MI symptoms, including: 1) Acute pulmonary oedema [7], defined as the presence of signs of pulmonary oedema, and need for treatment with nitrates or diuretics, 2) cardiogenic shock [7], defined as systolic BP $<90 \mathrm{mmHg}$ and/or diastolic $\mathrm{BP}<60 \mathrm{mmHg}$ associated with evidence of systemic hypo-perfusion (e.g. hyperlactatemia) and low cardiac output.

- Bradyarrythmia [7], requiring medical treatment or cardiac pacing.
- Peri-operative context [17], defined as MI occurring within the first $48 \mathrm{~h}$ after surgery.

- Coronary spasm [12], refers to a sudden, intense vasoconstriction of an epicardial coronary artery that causes vessel occlusion or near occlusion on coronary angiography, even in the absence of stimulation.

- Coronary embolism [7], defined as a high thrombus burden despite a relatively normal underlying vessel or recurrent coronary thrombus (left heart endocarditis, intracardiac mural thrombus, documented venous thrombus, and a patent foramen ovale or atrial septum defect).

- Acute infection [8], defined as a clinical diagnosis of acute infection by the physician, with at least one of the following: fever $>39^{\circ} \mathrm{C}$, tachypnea $>24$ breaths $/ \mathrm{min}$, tachycardia $>100$ beats $/ \mathrm{min}$, leukocytes $>12.10^{9} / \mathrm{L}$

- Supraventricular tachyarrhythmia (i.e. atrial fibrillation or flutter) lasting $\geq 20 \mathrm{~min}$ with a ventricular rate $>150$ beats/min [7].

- Ventricular tachycardia lasting $\geq 20 \mathrm{~min}$ [7].

- Convulsive seizure in the presence of generalized tonic- clonic seizure, regardless of its duration [18].

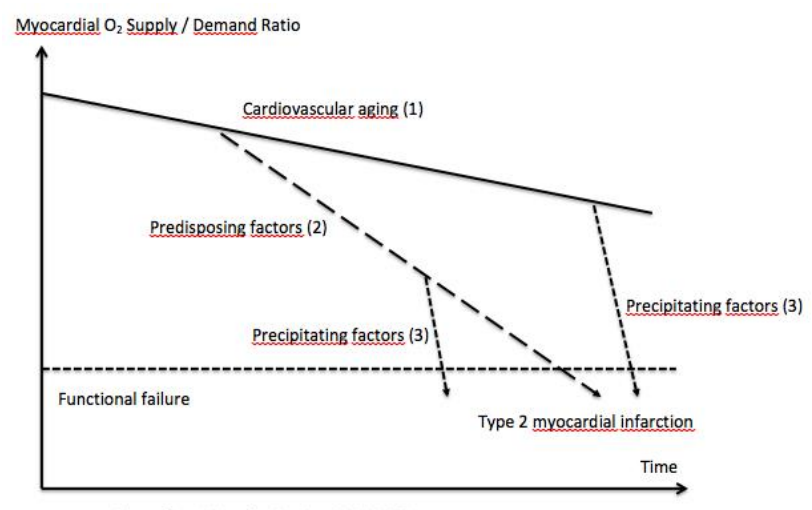

Adapted from JP Bouchen Rev Prat 1984, 34: 888

Figure 2. Geriatric model applied to type 2 myocardial infarction pathogenesis.

\section{Model definition}

Given the advanced age of patients with T2MI (median age $77 \mathrm{y}$ ) and their frequent comorbidities, we aimed to apply a geriatric functional model [19] to T2MI pathogenesis. In this model, functional failure (T2MI is here considered as an acute coronary failure) results from the superimposition of three factors:

1) Cardiovascular aging (mainly progression of coronary atherosclerosis), which per se is not responsible for functional failure whatever the age.

2) Predisposing factors related to chronic diseases (i.e. severe chronic anaemia, severe aortic stenosis, thyrotoxicosis, hypertensive cardiomyopathy). 
3) Acute potentially preventable triggering factors for decompensation (e.g. acute infection, tachyarrhythmia, acute respiratory failure).

Table 1. Patient characteristics (n (\%) or median [IQR]).

\begin{tabular}{|c|c|c|c|}
\hline & $\begin{array}{c}\text { Type } 1 \\
\text { n=3709 }\end{array}$ & $\begin{array}{l}\text { Type 2 } \\
n=863\end{array}$ & $\mathbf{p}$ \\
\hline \multicolumn{4}{|l|}{ Risk factors and comorbidities } \\
\hline Age, years & $67[56-79]$ & $77[65-84]$ & $<0.001$ \\
\hline Female & $971(26)$ & $382(44)$ & $<0.001$ \\
\hline BMI, $k g / m^{2}$ & 27 [24-30] & $26[23-29]$ & $<0.001$ \\
\hline Hypertension & $2151(58)$ & $645(75)$ & $<0.001$ \\
\hline Hypercholesterolemia & $1788(49)$ & $466(51)$ & 0.01 \\
\hline Family history of CAD & $1095(30)$ & $209(24)$ & $<0.001$ \\
\hline Smoking & $1167(32)$ & $143(17)$ & $<0.001$ \\
\hline Diabetes & $892(24)$ & $300(35)$ & $<0.001$ \\
\hline Chronic renal failure & $171(5)$ & $112(13)$ & $<0.001$ \\
\hline COPD & $243(7)$ & $128(15)$ & $<0.001$ \\
\hline Neoplasia & $476(13)$ & $179(21)$ & $<0.001$ \\
\hline \multicolumn{4}{|l|}{ Cardiovascular history } \\
\hline $\mathrm{CAD}$ & $831(23)$ & $322(37)$ & $<0.001$ \\
\hline Myocardial infarction & $319(9)$ & $135(16)$ & $<0.001$ \\
\hline Stroke & $252(7)$ & $119(14)$ & $<0.001$ \\
\hline PAD & $262(7)$ & $138(16)$ & $<0.001$ \\
\hline Heart failure & $135(4)$ & $113(13)$ & $<0.001$ \\
\hline Atrial fibrillation & $291(8)$ & $209(25)$ & $<0.001$ \\
\hline Aortic stenosis & $111(3)$ & $128(15)$ & $<0.001$ \\
\hline \multicolumn{4}{|l|}{ Chronic medication } \\
\hline ACEI/ARB & $1413(38)$ & $459(53)$ & $<0.001$ \\
\hline Beta blockers & $1075(29)$ & 409 (47) & $<0.001$ \\
\hline Statins & $1111(30)$ & $368(43)$ & $<0.001$ \\
\hline Diuretics & $986(27)$ & $461(53)$ & $<0.001$ \\
\hline Antiplatelet & $386(10)$ & $177(20)$ & $<0.001$ \\
\hline Aspirin & $935(25)$ & $346(40)$ & $<0.001$ \\
\hline VKA & $232(6)$ & $171(20)$ & $<0.001$ \\
\hline \multicolumn{4}{|l|}{ Clinical data at admission } \\
\hline Time SO-admission, $\min$ & $180[100-480]$ & $150[60-351]$ & $<0.001$ \\
\hline Heart rate, $b / \min$ & $77[66-90]$ & 85 [71-102] & $<0.001$ \\
\hline $\mathrm{SBP}, m m H g$ & $140[121-160]$ & $132[114-156]$ & $<0.001$ \\
\hline $\mathrm{DBP}, \mathrm{mmHg}$ & 80 [70-94] & $73[62-85]$ & $<0.001$ \\
\hline Anterior wall location & $1351(36)$ & $285(33)$ & 0.06 \\
\hline GRACE risk score & $143[120-170]$ & $171[138-197]$ & $<0.001$ \\
\hline Heart failure & $812(22)$ & $396(46)$ & $<0.001$ \\
\hline LVEF, \% & $55[45-60]$ & $50[40-60]$ & $<0.001$ \\
\hline \multicolumn{4}{|l|}{ ECG at admission } \\
\hline STEMI & $1940(52)$ & $209(24)$ & $<0.001$ \\
\hline $\mathrm{AF} /$ Flutter & $264(9)$ & $162(19)$ & $<0.001$ \\
\hline LBBB & $195(5)$ & $86(10)$ & $<0.001$ \\
\hline AVB & $91(2)$ & $22(1)$ & 0.003 \\
\hline \multicolumn{4}{|l|}{ Biological data } \\
\hline Hemoglobin, $g / 100 m L$ & $14.4[13-15.4]$ & $12.7[10.8-14.2]$ & $<0.001$ \\
\hline $\mathrm{CRP}, m g / L$ & $4.6[2.9-12.0]$ & $13.9[3.8-60.8]$ & $<0.001$ \\
\hline Creatinine, $\mu \mathrm{mol} / \mathrm{L}$ & $80[70-102]$ & $92[71-126]$ & $<0.001$ \\
\hline $\mathrm{GFR}, \mathrm{mL} / \mathrm{min} / 1.73 \mathrm{~m}^{2}$ & $67[54-108]$ & $54[36-80]$ & $<0.001$ \\
\hline LDL cholesterol, $g / L$ & $1.2[0.9-1.5]$ & $0.9[0.6-1.2]$ & $<0.001$ \\
\hline NT-proBNP, $p g / m L$ & $578[150-2334]$ & 3132 [729-10246] & $<0.001$ \\
\hline Troponin Ic peak, $\mu g / L$ & $17[3-68]$ & $3[1-14]$ & $<0.001$ \\
\hline Coronary angiography & 3709 (100) & $662(77)$ & $<0.001$ \\
\hline Normal coronary arteries & $10(0.3)$ & $144(22)$ & $<0.001$ \\
\hline MINOCA & $54(1)$ & $245(37)$ & $<0.001$ \\
\hline 3-vessel or left main disease & $1157(31)$ & $191(29)$ & 0.2 \\
\hline \multicolumn{4}{|l|}{ Revascularization } \\
\hline PCI & $2980(80)$ & $204(24)$ & $<0.001$ \\
\hline CABG & $229(6)$ & $40(5)$ & 0.1 \\
\hline Thrombolysis & $230(6)$ & $6(0.7)$ & $<0.001$ \\
\hline \multicolumn{4}{|l|}{ Hospital outcomes } \\
\hline ICU stay, days & $4[3-5]$ & $4[3-6]$ & 0.05 \\
\hline All cause death & $195(5)$ & $92(11)$ & $<0.001$ \\
\hline Cardiovascular death & $182(5)$ & $75(9)$ & $<0.001$ \\
\hline
\end{tabular}

ARB: angiotensin II receptor blocker; AVB: $2^{\text {nd }} / 3^{\text {rd }}$ degree atrio-ventricular block; BMI: body mass index; CAD: coronary artery disease; ACEI: Angiotensin converting enzyme inhibitor; CABG: coronary artery bypass grafting; $\mathrm{CK}$ : creatine kinase; COPD: chronic obstructive pulmonary disease; CRP: C-reactive protein;
DBP: diastolic blood pressure; GFR: estimated glomerular filtration rate; ICU: intensive care unit; IQR: Interquartile range; LBBB: left bundle branch block; LVEF: left ventricular ejection fraction; MINOCA: myocardial infarction with non-obstructive coronary arteria; NT-proBNP: N-Terminal pro Brain Natriuretic Peptide; PAD: peripheral arterial disease; PCI: percutaeous coronary intervention; SBP: systolic blood pressure; SO: Symptom onset; STEMI: ST segment elevation myocardial infarction; VKA: vitamin K antagonist; AVB: atrioventricular block.

Both predisposing and precipitating factors, either combined or separate, may be responsible for functional failure. This model is presented in Figure 2.

\section{Statistical analyses}

Continuous variables were expressed as mean \pm standard deviation or median and interquartile range. A Kolmogorov-Smirnov test was performed to analyse the normality of continuous variables. Student's t-test or the Mann-Whitney test was used to compare continuous variables. Chi 2 or the Fisher's test was used to compare dichotomous data. The threshold for significance was set at 5\%. SPSS version 12.0.1 (IBM Inc, USA) was used for all statistical analysis.

\section{RESULTS}

\section{Baseline characteristics}

Among the 4572 patients, $3710(81 \%)$ had T1MI and 862 had T2MI (19\%) (Table 1). Overall, T2MI patients were 10 years older (median age 77 [65-84] vs 67 [56-79] y, $\mathrm{p}<0.001)$, more frequently female ( $44 \%$ vs $26 \%$, p $<0.001$ ) had more comorbidities and cardiovascular history (i.e. coronary artery disease: $37 \%$ vs $23 \%, \mathrm{p}<0.001$ ) and subsequently more chronic cardiovascular treatments (i.e. anticoagulants or antiplatelet medications) than T1MI patients. Acute clinical presentation was more severe in T2MI (i.e. heart failure: $46 \%$ vs $22 \%, \mathrm{p}<0.001$ and GRACE risk score 171 [138-197] vs 143 [120-170], $\mathrm{p}<0.001)$. ECG at admission showed less frequent ST segment elevation $(24 \%$ vs $52 \%, \mathrm{p}<0.001)$ and a higher rate of atrial fibrillation or flutter $(19 \%$ vs $9 \%, \mathrm{p}<0.001)$. Troponin Ic peak was much lower (3 [1-14] vs 17 [3-68] $\mu \mathrm{g} / \mathrm{L}$ ), although NT-proBNP level was markedly higher (3132 [729-10246] vs 578 [150-2334] pg/mL) in T2MI patients. Troponin peaks remained lower in T2MI group compared with T1MI group for both ST-segment elevation MI (STEMI) (respective median troponin Ic peak: 9.6 vs $49 \mu \mathrm{g} / \mathrm{L}, \mathrm{p}<0.001)$ and non STEMI patients (2.7 vs $4.9 \mu \mathrm{g} / \mathrm{L}, \mathrm{p}<0.001)$ Median time from symptoms onset to admission was 30 min shorter in T2MI patients $(p<0.001)$. Coronary angiography was more common in cases of T1MI $(\mathrm{p}<0.001)$. Among patients with angiography data, MINOCA accounted for $37 \%$ of T2MI versus $1 \%$ of T1MI. Three-vessel or main left disease was similar for T1MI and T2MI groups. Revascularization 
procedures were used in 30\% of T2MI patients and coronary artery bypass surgery rate was similar for both groups. All-cause and cardiovascular in-hospital deaths were twice as frequent among T2MI patients (11\% vs $5 \%$ and $9 \%$ vs $5 \%$, respectively, $\mathrm{p}<0.001$ ).

Multivariate analysis of factors associated with T2MI versus T1MI is presented in figure 3. Acute heart failure, C-reactive protein elevation and tachycardia were associated with a higher risk of T2MI whereas chest pain, high Troponin I peak and ST-segment elevation were associated with a higher risk of T1MI. Older patients, women, and patients with chronic heart failure or diabetes were groups at higher risk of T2MI; patients with chronic kidney disease tend to have a higher risk of T1MI.

\section{T2MI Etiologic analysis}

Among the 862 cases of T2MI, 208 predisposing and 764 precipitating situations were identified with the prospective adjudication procedure (Fig. 4):
The main predisposing factors were chronic severe anaemia (10\%) and severe aortic stenosis (7\%).

Acute infection was by far the most common acute precipitating factor (39\%), and mainly included respiratory infection $(26 \%)$, followed by supraventricular tachyarrhythmia (13\%) and acute heart failure (10\%).

Main characteristics of T2MI patients with predisposing thyrotoxicosis or presenting an acute infection, acute heart failure, or convulsive seizures as T2MI trigger are presented in table 2.

Interestingly, $122(14 \%)$ patients had combined chronic and acute conditions for T2MI. Among predisposing factors, chronic severe anaemia and severe aortic stenosis were the most frequent factors combined with acute triggers, which were mainly infection and acute heart failure. The main associations between etiological factors are shown in Figure 5.

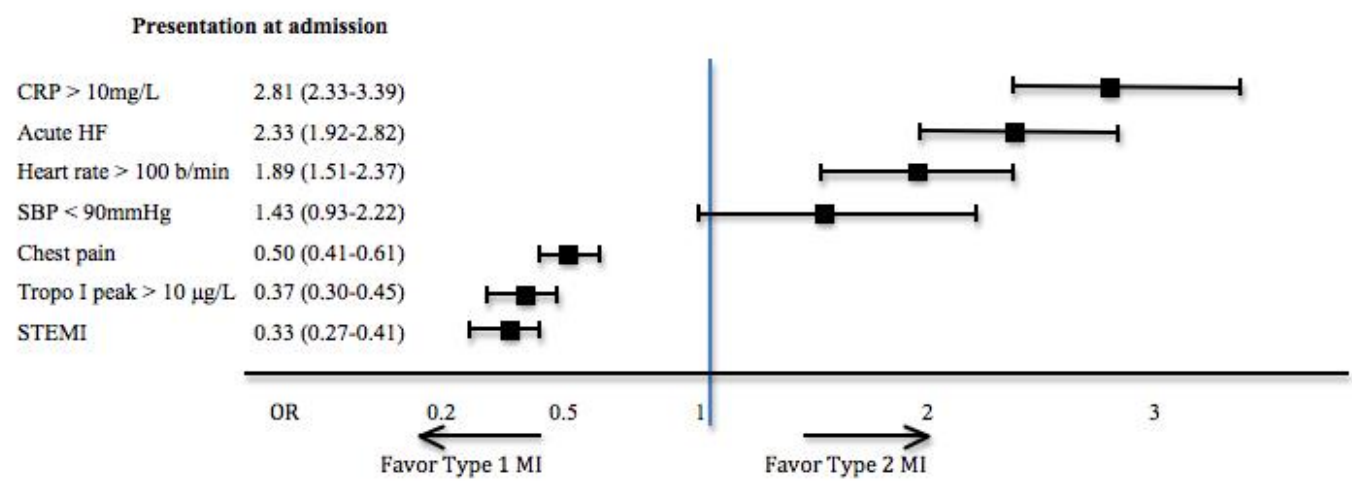

Risk factors and comorbidities

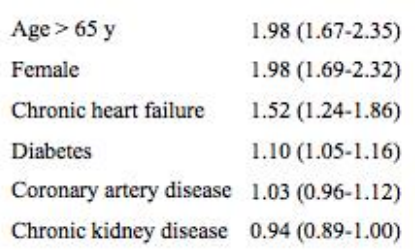

OR $\quad \sum_{\text {Favor Type } 1}^{0.2} \mathrm{MI}$

Figure Multivariate analysis of factors associated with type 2 myocardial infarction. CRP: Creactive protein; $\mathrm{HF}$ : heart failure; MI: myocardial infarction, SBPL systolic blood pressure; STEMI: ST-segment elevation myocardial infarction; Tropo I peak: cardiac troponin I peak.

\section{DISCUSSION}

The present study is to date one of the largest real-life studies to comprehensively analyse the whole spectrum of clinical features for T2MI patients, using a comprehensive geriatric approach of pathogenesis. To our knowledge, only Saaby et al. have clearly proposed a set of etiological criteria and cut-offs for T2MI adjudication, reflecting an imbalance between the supply and demand of myocardial oxygen [7]. However, our large database allows a wider evaluation of less frequent clinical situations, as initial restrictive criteria could have led to an under-evaluation of T2MI frequency. T2MI was identified in $7.3 \%$ of patients with elevated troponins in an emergency setting 
[9], which is markedly lower than other studies for which T2MI adjudication was at the experts' discretion [20]. A more comprehensive and reproducible approach is needed to simplify the diagnosis procedure for clinicians and to harmonise research. Using the French geriatric model of Bouchon [19], we propose here a new approach for T2MI pathogenesis, considering T2MI as a functional cardiovascular failure, resulting from age, combining chronic predisposing and acute conditions for decompensation. Data from a large validated MI survey and a systematic adjudication procedure were used to test this model. Moreover, our study extends T2MI pathogenesis to additional factors, which may contribute to myocardial supply/demand mismatch.

Table 2. Main characteristics (n (\%) or median [IQR]) of patients with type 2 myocardial infarction and acute infection, heart failure, thyrotoxicosis or seizure.

\begin{tabular}{|c|c|c|c|c|}
\hline & $\begin{array}{l}\text { Infection } \\
(\mathbf{n}=335)\end{array}$ & $\begin{array}{l}\text { Heart failure } \\
(\mathrm{n}=86)\end{array}$ & $\begin{array}{l}\text { Thyrotoxicosis } \\
(n=28)\end{array}$ & $\begin{array}{l}\text { Seizure } \\
(n=14)\end{array}$ \\
\hline Age & $79[69-85]$ & $79[65-85]$ & $80[72-84]$ & $66[57-85]$ \\
\hline STEMI & $93(27.8)$ & $24(27.9)$ & $7(25)$ & $3(21.4)$ \\
\hline Troponin peak, $\mu g / L$ & $4.7[1.2-15.2]$ & $5.8[1.8-21]$ & $7.1[0.9-17.7]$ & $2.2[72-84]$ \\
\hline LVEF, \% & $45[35-55]$ & $35[25-45]$ & $55[30-63]$ & $40[40-60]$ \\
\hline Heart Rate, $b / \min$ & 86 [73-102] & 88 [73-110] & 95 [75-117] & $92[79-100]$ \\
\hline SBP, $\mathrm{mmHg}$ & $131[115-154]$ & $123[90-161]$ & $130[111-150]$ & 120 [101-145] \\
\hline $\mathrm{DBP}, \mathrm{mmHg}$ & $72[61-84]$ & $69[54-82]$ & $75[65-88]$ & $65[56-84]$ \\
\hline Coronary angiography & $235(70.1)$ & $61(70.9)$ & $21(75)$ & $14(100)$ \\
\hline Normal & $25(10.6)$ & $10(16.4)$ & $1(4.8)$ & $7(50)$ \\
\hline MINOCA & $46(19.5)$ & $22(36.1)$ & $2(8.5)$ & $10(71.4)$ \\
\hline 3 vessel / left main disease & $92(39.1)$ & $20(32.8)$ & $9(42.9)$ & $2(14.3)$ \\
\hline PCI & $86(25.7)$ & $18(20.9)$ & $12(42.9)$ & $2(14.3)$ \\
\hline In-hospital death & $38(11.3)$ & $24(27.9)$ & $3(10.7)$ & $1(7.1)$ \\
\hline
\end{tabular}
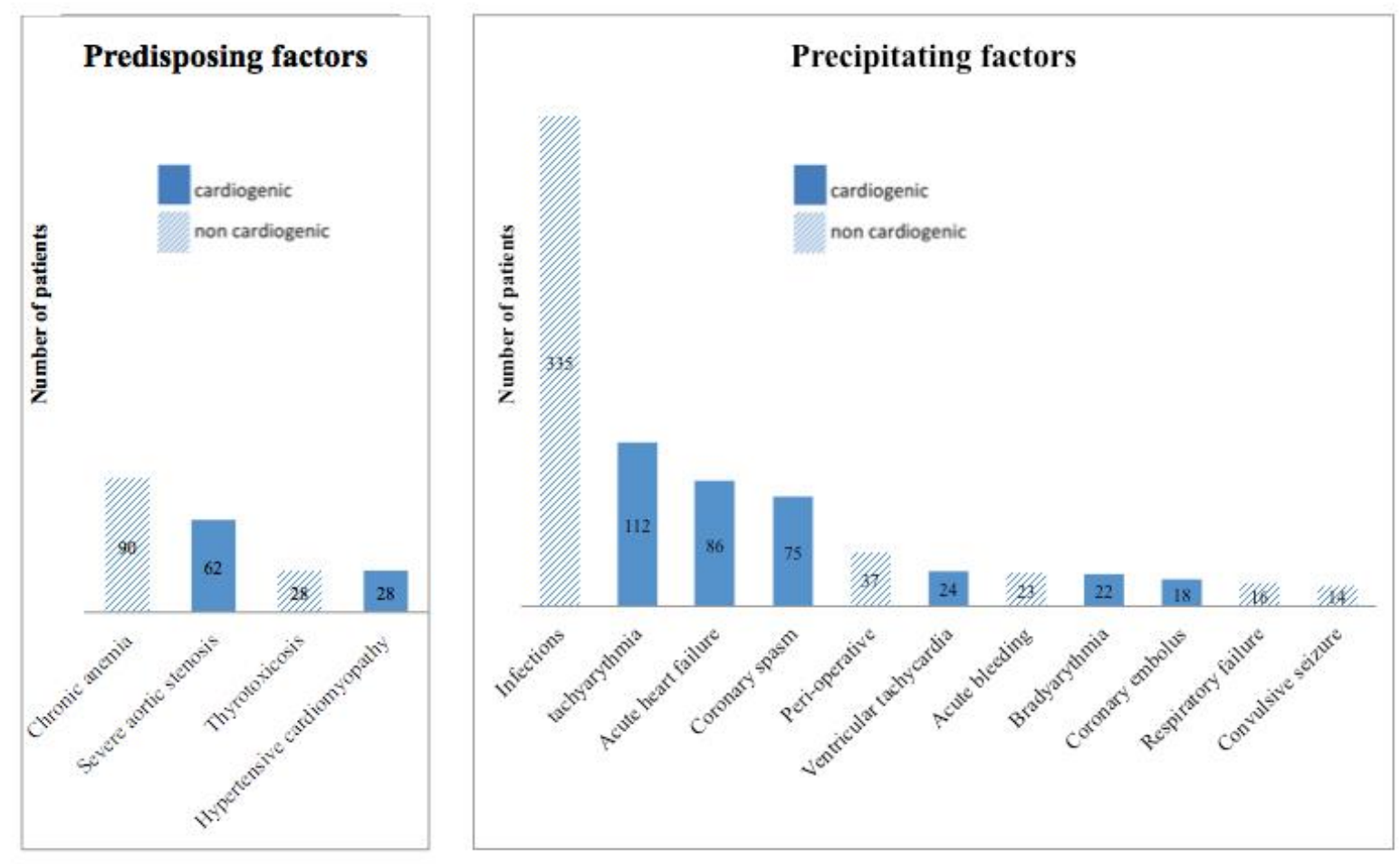

Figure 4. Predisposing and precipitating factors for type 2 myocardial infarction. 
The main results are as follows:

- Chronic anaemia and severe aortic stenosis are chronic situations at risk for T2MI, in particular when associated with acute infections or tachyarrhythmia.

- The leading acute decompensating factor for T2MI was non-cardiogenic, i.e. infection and particularly respiratory tract infection,

- Thyrotoxicosis, regardless of tachyarrhythmia, emerged as a major underlying mechanism, exhibiting the same prevalence as hypertensive cardiomyopathy.

Here, the characteristics of T1MI and T2MI and their relative proportions are consistent with previous studies. T2MI patients were 10 year older, more often women, had more cardiovascular risk and comorbidities than patients with T1MI $[8,9,18]$. Moreover, troponin Ic peak was lower [20], and ST segment elevation was much less frequent (24\%) [10]. In contrast, rhythm and conduction disorders, including atrial fibrillation and left bundle branch block were more frequent [21]. Patients with T2MI had higher GRACE scores than T1MI patients, and inhospital mortality was twice as high [10]. Surprisingly, time from symptoms onset to admission was shorter for T2MI patients, despite the less frequency of ST segment elevation. One explanation could be the more severe clinical presentation, especially the more frequent acute heart failure, resulting in a faster alerting time and a prompter medical support.

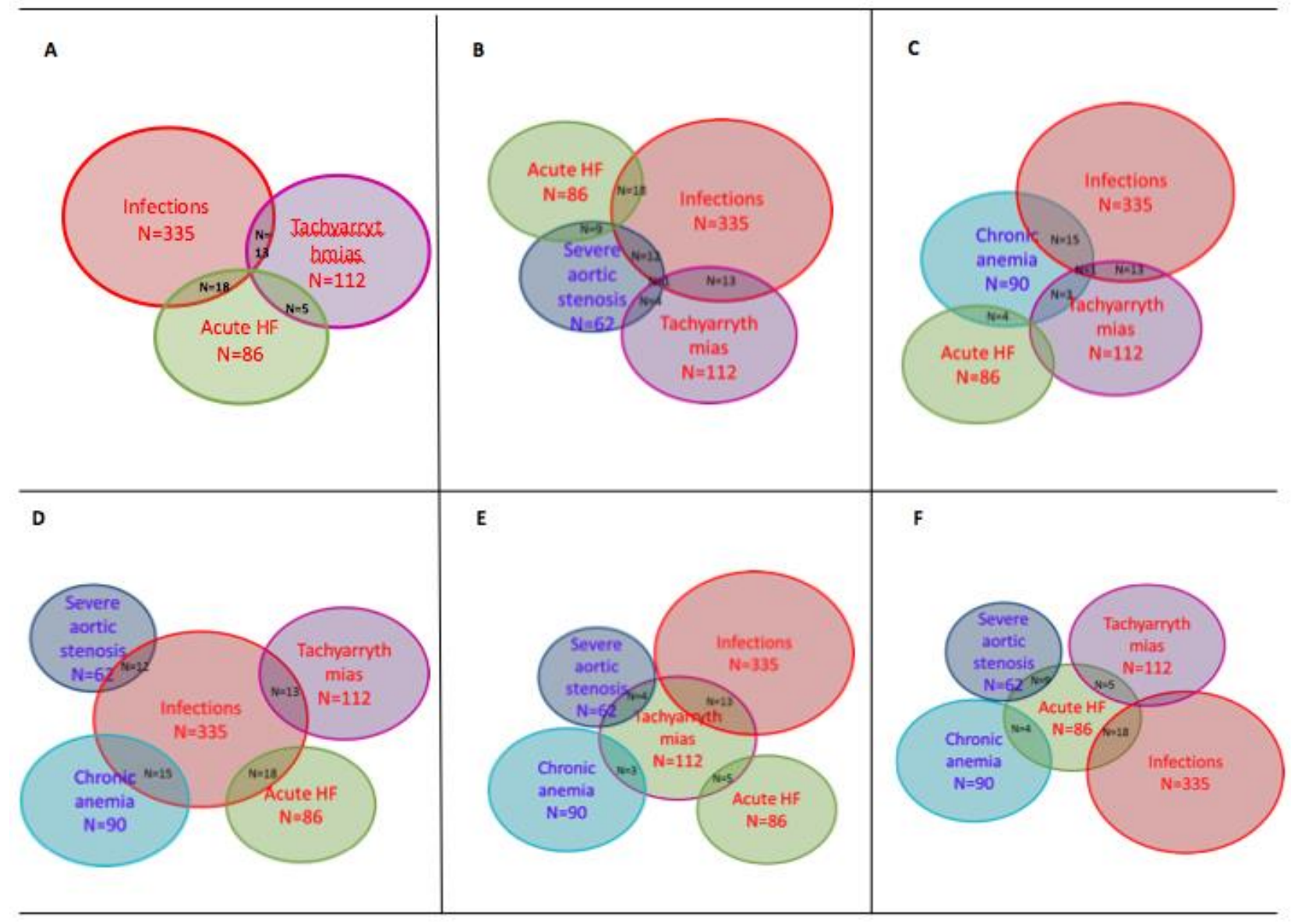

Figure 5. Main combinations of etiologic factors for type 2 acute myocardial infarction. (A) Combination of acute factors. (B and C) Combinations of acute and chronic factors. (D) Acute and chronic factors combined with infection. (E) Acute and chronic factors combined with tachyarrhythmia. F. Acute and chronic factors combined with acute heart failure.

Our findings are also consistent with retrospective studies for some of the leading causes of T2MI (i.e. tachy/brady-arrhythmias, severe aortic stenosis, hypertensive cardiomyopathy, cardiogenic shock, severe respiratory failure, severe anaemia, coronary spasm, and coronary embolism) which have been widely described, and are included as part of the initial definition [12] and confirmed in the new definition [1]. Notably, only patients with definite MI criteria (i.e. troponin elevation with chest pain and/or ECG changes) were considered in this study even though T2MI causes mentioned above are also frequently responsible for myocardial injury without 
infarction (i.e. troponin elevation without such signs), named non-ischemic myocardial injury (NIMI) in the new universal definition of MI [1]. The distinction between T2MI and NIMI is often difficult in clinical practice but appears to be of low prognostic value [20]. To date, the therapeutic strategy of T2MI and NIMI has still to be determinated [1].

Anaemia, a widespread condition in the elderly, was one of the leading causes of T2MI in the literature $[9,10,21]$. Our findings reveal that severe anaemia was the main predisposing factor (10\%) and was often combined with other contributing factors. Therefore, patients with severe anaemia are at increased risk for T2MI, in particular if there is concomitant infection (17\%) or, more rarely, acute heart failure or tachyarrhythmia. However, acute bleeding and chronic anaemia should be categorized separately, as patient characteristics, myocardial tolerance and prognosis largely differ [22]. In older anaemic patients, an association between red blood cell transfusion in the acute phase of MI and decreased mortality was recently highlighted [23], suggesting the potential benefit of transfusion following anaemia in T2MI.

Acute heart failure was the second acute cardiogenic precipitating factor, often associated with infections (21\%) and chronic conditions such as anemia or severe aortic stenosis.

Surprisingly, even though it is the most frequent cause of T2MI in a number of studies [5] including our own, infection was not reported as an etiologic factor in the $4^{\text {th }}$ Universal Definition of MI [1]. Notably, we observed that infection was the acute precipitating factor the most often associated with other contributing factors, i.e. either chronic (anaemia and severe aortic stenosis) or acute (acute HF or tachyarrhythmia) conditions. Moreover, our data show that infections of the respiratory tract are the infection type at higher risk for T2MI. However, we found that other types of sepsis, including urinary tract infections, are also potential triggering mechanisms for T2MI, and this hypothesis has also been put forward for T1MI [24]. Infection could prompt both a decrease in $\mathrm{O}_{2}$ supply via hypoxemia and an increase in myocardial consumption of $\mathrm{O}_{2}$ because of sepsis-related high cardiac output [25]. This could explain in part the increased hibernal incidence of MI [24] and the protective effect of influenza [26] and pneumococcal [27] vaccinations.

We have observed additional causes that were less known and have never been specifically addressed in such large series of cases. Thyrotoxicosis has only been previously reported in 4 patients by Stein et al. [10]. Our work shows that overt hyperthyroidism, although very rare in the community-dwelling older population [28], is the second leading non-cardiogenic predisposing factor, behind anaemia. Myocardial ischemia has already been linked to an increase in myocardial oxygen demand in response to the increase in myocardial contractility and workload associated with elevated thyroid hormone levels (i.e. elevated T4, T3, or both) [29].

Hyperthyroidism has also been shown to cause severe, diffuse left-sided coronary vasospasm resulting in MI and/or fatal arrhythmia, especially in young females, even in the absence of coronary artery disease [30]. Interestingly, successful treatment of hyperthyroidism has been associated with a reversal of these symptoms [29].

Convulsive seizure, a rare acute condition (2\%) preceding T2MI in the present study, has been scarcely described, as case reports, in both T1MI [31] and T2MI $[18,31]$. A massive catecholamine release, similar to what is observed in Takotsubo cardiomyopathy induced by ischemic stroke or epileptic events [32], could explain an increased myocardial oxygen demand, as shown by the concomitant tachycardia [31].

Peri-operative T2MI has only rarely been described. Notably, a retrospective review reported that non-cardiac surgery was the most common associated setting for T2MI or myocardial injury (38\%) [33]. In contrast, our prospective study reports a lower prevalence -at 5\%- of precipitating conditions among patients hospitalized in cardiology ICU. While T1MI in the post-operative setting is rare, myocardial oxygen supply/demand balance can be altered [17]. Post-operative T2MI most likely has multiple pathophysiological pathways, including tachycardia, acute bleeding, and hypoxemia.

Severe aortic stenosis has been associated with T2MI $[2,10,33]$. In the present study, this chronic predisposing factor was the second most frequent predisposing factor of T2MI, and was often associated with acute conditions (infections, acute heart failure or tachyarrhythmia). Experimental works in patients with severe aortic stenosis undergoing aortic balloon valvuloplasty have shown that hemodynamic stress decreases aortic pressure (thus decreasing coronary perfusion and oxygen supply) and increases left ventricular pressure (increasing oxygen demand) [34]. Further studies are needed to fully understand the pathophysiological link between aortic stenosis and acute situations at risk of MI triggering.

\section{Study limitations}

The present study has several limitations. First, this work was limited to patients hospitalized in cardiology ICU, and is thus difficult to extrapolate to other hospital departments where T2MI incidence and characteristics may be different [7]. Secondly, even if coronary angiography was available for a large proportion of patients, this examination has a weak sensibility for small or eccentric thrombus. Coronary plaque rupture and ulceration could thus have been under-diagnosed, and no 
other imaging techniques, such as intravascular ultrasound, were used, resulting in potential misclassifications of T2MI [5]. Third, some of the criteria for T2MI adjudication are debatable, including severe anaemia. Because anaemia is a widespread condition in older adults, we deliberately choose a restrictive haemoglobin cut-off in order to avoid overestimation chronic anaemia burden, which has been done in past studies as well [7]. Fourth, since the beginning of the study, a change in MI definition has occurred [1,12]. Notably, myocardial injury has been clearly distinguished from MI by the fourth universal definition. However, only patients with clinical, imaging or ECG signs of myocardial ischemia are included in the RICO survey, which corresponds to the new definition of MI, and nonischemic myocardial injury have thus not been included in this study. Fifth, several criteria of T2MI, including acute infections, have not been acknowledged as pathophysiological factors by the 3rd and 4th universal definitions. Consistently with previous studies, we believe that those criteria are however responsible for an ischaemic imbalance of oxygen, defined as the pathophysiological pattern of T2MI by $4^{\text {th }}$ the universal definition. Finally, despite the rigorous use of systematic diagnostic criteria for each case by two expert investigators, the clinical situations leading to T2MI are probably too polymorphic and multifactorial to be reduced to specific criteria. Because our model does not aim to be exhaustive, the frequency of T2MI has probably been underestimated.

\section{Conclusions}

T2MI stems from a number of heterogeneous underlying factors, including age-related cardiovascular aging. In our large population-based study, we used a prospective, comprehensive and analytic strategy to characterize patients with T2MI hospitalized in cardiology ICU. We identified older age, acute heart failure, tachycardia and C-reactive protein elevation as associated with T2MI, whereas chest pain, STEMI and high troponin elevation were more frequent in T1MI. Our findings have led to propose a new model for T2MI, based on a geriatricderived pathogenesis and including chronic and acute conditions, which can be superposed in some cases. We showed that respiratory infections are by far the most common acute decompensating factor, as they were found in more than a quarter of T2MI patients. Among predisposing factors, severe anaemia and aortic stenosis are the leading contributors. The characterization of contributing factors and their mutual interactions is a key step for understanding myocardial oxygen imbalance and improving the management of T2MI, frequently encountered in older frail patients. Our findings may lead to the development of personalized treatment strategies and better outcomes for T2MI patients.

\section{Acknowledgments}

The authors thank Suzanne Rankin for the English review of the manuscript, Florence Bichat, Morgane Lainé, Maud Maza, for technical assistance. This work was supported by the University Hospital of Dijon, the Association de Cardiologie de Bourgogne, and by grants from the Agence Régionale de Santé (ARS) de Bourgogne Franche Comté, Institut National de la Santé et de la Recherche Médicale (INSERM) and from the Conseil Régional de Bourgogne Franche Comté.

\section{Conflict of interest statement}

The authors have no conflict of interest to disclose inconnection with the present paper.

\section{References}

[1] Thygesen K, Alpert JS, Jaffe AS, Chaitman BR, Bax JJ, Morrow DA, et al (2018). Fourth Universal Definition of Myocardial Infarction (2018). J Am Coll Cardiol, 72(18): 2231-2264.

[2] Friedberg CK, Horn H (1939). Acute myocardial infarction not due to coronary artery occlusion. J Am Med Assoc, 112(17): 1675-1679.

[3] Crea F, Libby P (2017). Acute Coronary Syndromes: The Way Forward From Mechanisms to Precision Treatment. Circulation, 136(12): 1155-1166.

[4] Alpert JS, Thygesen KA, White HD, Jaffe AS (2014). Diagnostic and Therapeutic Implications of Type 2 Myocardial Infarction: Review and Commentary. Am J Med, 127(2): 105-108.

[5] Sandoval Y, Thygesen K (2017). Myocardial Infarction Type 2 and Myocardial Injury. Clin Chem, 63(1): 101107.

[6] Gaggin HK, Liu Y, Lyass A, Van Kimmenade RRJ, Motiwala SR, Kelly NP, et al (2017). Incident Type 2 Myocardial Infarction in a Cohort of Patients Undergoing Coronary or Peripheral Arterial Angiography. Circulation, 135(2): 116-127.

[7] Saaby L, Poulsen TS, Hosbond S, Larsen TB, Pyndt Diederichsen AC, Hallas J, et al (2013). Classification of Myocardial Infarction: Frequency and Features of Type 2 Myocardial Infarction. Am J Med, 126(9): 789797.

[8] Landes U, Bental T, Orvin K, Vaknin-Assa H, Rechavia E, Iakobishvili Z, et al (2016). Type 2 myocardial infarction: A descriptive analysis and comparison with type 1 myocardial infarction. J Cardiol, 67(1): 51-56.

[9] Saaby L, Poulsen TS, Diederichsen ACP, Hosbond S, Larsen TB, Schmidt H, et al (2014). Mortality Rate in Type 2 Myocardial Infarction: Observations from an 
Unselected Hospital Cohort. Am J Med, 127(4): 295302.

[10] Stein GY, Herscovici G, Korenfeld R, Matetzky S, Gottlieb S, Alon D, et al (2014). Type-II Myocardial Infarction - Patient Characteristics, Management and Outcomes. PLoS ONE, 9(1): e84285.

[11] Zeller M, Steg PG, Ravisy J, Lorgis L, Laurent Y, Sicard P, et al (2008). Relation between body mass index, waist circumference, and death after acute myocardial infarction. Circulation, 118(5): 482-490.

[12] Thygesen K, Alpert JS, Jaffe AS, Simoons ML, Chaitman BR, White HD, et al (2012). Third universal definition of myocardial infarction. Eur Heart J, 33(20): 2551-2567.

[13] Granger CB, Goldberg RJ, Dabbous O, Pieper KS, Eagle KA, Cannon CP, et al (2003). Predictors of hospital mortality in the global registry of acute coronary events. Arch Intern Med, 163(19): 23452353.

[14] Arrebola MM, Lillo JA, Diez De Los Ríos MJ, Rodríguez M, Dayaldasani A, Yahyaoui R, Pérez V (2010). Analytical performance of a sensitive assay for cardiac troponin I with loci technology. Clin Biochem, 43(12): 998-1002.

[15] Pasupathy S, Tavella R, Beltrame JF (2017). Myocardial Infarction With Nonobstructive Coronary Arteries (MINOCA): The Past, Present, and Future Management. Circulation, 135(16): 1490-1493.

[16] 2006 WRITING COMMITTEE MEMBERS, Bonow RO, Carabello BA, Chatterjee K, De Leon AC, Faxon DP, et al (2008). 2008 Focused Update Incorporated Into the ACC/AHA 2006 Guidelines for the Management of Patients With Valvular Heart Disease: A Report of the American College of Cardiology/American Heart Association Task Force on Practice Guidelines (Writing Committee to Revise the 1998 Guidelines for the Management of Patients With Valvular Heart Disease): Endorsed by the Society of Cardiovascular Anesthesiologists, Society for Cardiovascular Angiography and Interventions, and Society of Thoracic Surgeons. Circulation, 118(15): e523-e661.

[17] Landesberg G, Beattie WS, Mosseri M, Jaffe AS, Alpert JS (2009). Perioperative Myocardial Infarction. Circulation, 119(22): 2936-2944.

[18] Park J, Shin JH, Kim SH, Lim YH, Lee JU, Kim KS, et al (2011). Type 2 Myocardial Infarction Following Generalized Tonic-Clonic Seizure. Korean Circ J, 41(11): 681.

[19] Bouchon J-P (1984). $1+2+3$ ou comment tenter d'être efficace en gériatrie? Rev Prat, 34:888-892

[20] Putot A, Derrida SB, Zeller M, Avondo A, Ray P, Manckoundia P, Cottin Y (2018). Short-Term Prognosis of Myocardial Injury, Type 1 and Type 2 Myocardial Infarction in the Emergency Unit. Am J Med, 131(10): 1209-1219.

[21] Baron T, Hambraeus K, Sundström J, Erlinge D, Jernberg T, Lindahl B, TOTAL-AMI study group
(2015). Type 2 myocardial infarction in clinical practice. Heart, 101(2): 101-106.

[22] Ducrocq G, Puymirat E, Steg PG, Henry P, Martelet M, Karam C, et al (2015). Blood transfusion, bleeding, anemia, and survival in patients with acute myocardial infarction: FAST-MI registry. Am Heart J, 170(4): 726734.e2.

[23] Putot A, Zeller M, Perrin S, Beer JC, Ravisy J, Guenancia C, et al (2017). Blood Transfusion in Elderly Patients with Acute Myocardial Infarction: Data From the RICO Survey. Am J Med, 131(4):422-429.e4.

[24] Smeeth L, Thomas SL, Hall AJ, Hubbard R, Farrington P, Vallance P (2004). Risk of myocardial infarction and stroke after acute infection or vaccination. $\mathrm{N}$ Engl $\mathrm{J}$ Med, 351(25): 2611-2618

[25] Mihatov N, Januzzi JL, Gaggin HK (2017). Type 2 myocardial infarction due to supply-demand mismatch. Trends Cardiovasc Med, 27(6): 408-417.

[26] Ciszewski A, Bilinska ZT, Brydak LB, Kepka C, Kruk M, Romanowska M, et al (2008). Influenza vaccination in secondary prevention from coronary ischaemic events in coronary artery disease: FLUCAD study. Eur Heart J, 29(11): 1350-1358.

[27] Vlachopoulos CV, Terentes-Printzios DG, Aznaouridis KA, Pietri PG, Stefanadis CI (2015). Association between pneumococcal vaccination and cardiovascular outcomes: a systematic review and meta-analysis of cohort studies. Eur J Prev Cardiol, 22(9): 1185-1199.

[28] Cappola AR, Fried LP, Arnold AM, Danese MD, Kuller LH, Burke GL, et al (2006). Thyroid Status, Cardiovascular Risk, and Mortality in Older Adults: The Cardiovascular Health Study. JAMA J Am Med Assoc, 295(9): 1033-1041.

[29] Klein I, Danzi S (2007). Thyroid disease and the heart. Circulation, 116(15): 1725-1735.

[30] Lee SY, Yu CW, Choi YJ, Choi RK, Park JS, Lee HJ, et al (2014). A comparison of clinical features of coronary artery spasm with and without thyrotoxicosis. Coron Artery Dis, 25(2): 125-132.

[31] Chin PS, Branch KR, Becker KJ (2004). Myocardial infarction following brief convulsive seizures. Neurology, 63(12): 2453-2454.

[32] Blanc C, Zeller M, Cottin Y, Daubail B, Vialatte AL, Giroud M, Béjot Y (2015). Takotsubo Cardiomyopathy Following Acute Cerebral Events. Eur Neurol, 74(3-4): 163-168.

[33] Smilowitz NR, Weiss MC, Mauricio R, Mahajan AM, Dugan KE, Devanabanda A, et al (2016). Provoking conditions, management and outcomes of type 2 myocardial infarction and myocardial necrosis. Int $\mathbf{J}$ Cardiol, 218: 196-201.

[34] Smucker ML, Tedesco CL, Manning SB, Owen RM, Feldman MD (1988). Demonstration of an imbalance between coronary perfusion and excessive load as a mechanism of ischemia during stress in patients with aortic stenosis. Circulation, 78(3): 573-582. 